\title{
Biodiversity of Relict Vascular Plants in Bulgaria
}

\author{
Dimcho Zahariev \\ Faculty of Natural Sciences, University of Shumen Bishop Konstantin Preslavski \\ 115 Universitetska Str., 9712 Shumen, Bulgaria \\ dimtchoz@abv.bg
}

\begin{abstract}
Climate changes observed in the last years pose a serious threat to biodiversity. Similar climatic changes, nevertheless, have occurred many times in our planet's history. Relict plants that survived after experiencing climate change can give us information about the past and the future of species. The rich biodiversity in the countries of Southern Europe, including Bulgaria, is shaped by a large number of relict plants. To date, the biodiversity of relict plants in Bulgaria has not been systematically described and remains somewhat unknown. Our aim is to systematize available information and present biodiversity of relict vascular plants in Bulgaria. Using a critical approach, we discovered 346 species of 207 genera and 81 families of relict origin. This number accounts for $8.74 \%$ of the natural flora of Bulgaria and $8.43 \%$ of the total flora of Bulgaria (which includes foreign species). We divided relict plants into two groups: tertiary relicts (183 species) and quaternary relicts (163 species). The quaternary relicts we divided into 3 groups: glacial relicts (143 species), interglacial relicts (13 species) and postglacial relicts ( 7 species). Among the relicts with the largest number are perennial herbaceous plants, followed by shrubs and trees. 144 relict species have conservation status.
\end{abstract}

Keywords: relict, vascular plants, tertiary, quaternary, glacial, interglacial, postglacial

\section{INTRODUCTION}

One problem of a global scale today is the loss of species and the rapid impoverishment of ecosystems. Paleontology has sufficient evidence that the extinction of species and even of large taxonomic groups has occurred throughout the history of our Earth. However, we should not remain calm in the face of the facts. In the geological past, these processes were caused by natural changes in the landscape, the atmosphere, and the climate. Today, a large number species is endangered by the expansion of human activity. While current and past changes are comparable in their scale, the speed of the changes that take place accelerates. The solution of this problem should be found in the joint efforts of experts from all fields of human knowledge and the economy. One way for biologists to effectively contribute to solving the problem is by focusing their research on relicts. To this end, it is important for us to study the origin, the history and the current condition of relicts.

The term "relict" was introduced to Biology (in particular to Zoology) by Lovén [1] to explain findings of marine-type mysid crustaceans in freshwater lakes. The ecological and geographical criteria of recent relicts were analyzed in detail by Birshstein [2]. According to this author, those taxa that have remained almost unchanged in time (or are characterized by a delayed rate of evolution) are considered relict taxa. Vulf [3] defines "relict species" as remnants of more or less ancient flora, which occupied a relict area. The relict area is a remnant of a larger area, which was formed under specific conditions in the past different from those of other areas. In this sense, the term "relict species" is a historical-geographical concept, associated with the historical range of the species. The age of the relicts is determined by the time when the species has become a part of the flora composition.

The origin and distribution of relicts can often be linked to past changes in their environment, in particular climate changes. Therefore, they can be called "climate relicts". The status of a species as climate relict is usually determined on biogeographical basis, using current geographical distribution and attachment to specific habitats. Most often climate relicts were separated into three groups: 1 . Tertiary relicts that withdrew with the onset of drier and cooler climatic conditions during the late Tertiary and early Quaternary; 2. Glacial relicts that are more widely distributed during Quaternary cold stages and have experienced significant range contractions since the LGM; 3. Postglacial relicts that have survived to this day in regions with warmer climates, such as in the early Holocene [4]. 
In this article, we will use the categorization of relict species into three groups as suggested by Hampe \& Jump [4] and most widely used in botanical literature. On this basis, we will divide the plant relicts into two main groups: tertiary relicts and quaternary relicts. We will then divide quaternary relicts into 3 groups: glacial, interglacial and postglacial. We believe that this division most accurately reflects the origin of the relicts according to geological and related climate change.

In order to understand this categorization, we will provide a brief overview of the climate in the Cenozoic era, the time, in which these relict species originated. It is believed that the Cenozoic era began after the extinction of the dinosaurs before ca. 65.5 million years and continues to this day. Cenozoic (Neozoic, Cainozoic) era is divided into three periods: Paleogene (with three epochs: Paleocene, Eocene and Oligocene), Neogene (with two epochs: Miocene and Pliocene) and Quaternary (with two epochs: Pleistocene and Holocene). The first two periods in the past have been designated as Tertiary, a term that is still used in biogeography. During Paleogene and Neogene temperatures and the $\mathrm{CO}_{2}$ content were higher than those today. The climate was warm and humid. During these periods, there was a gradual cooling, which culminated in the third period, the Quaternary (Fig. 1) [5].

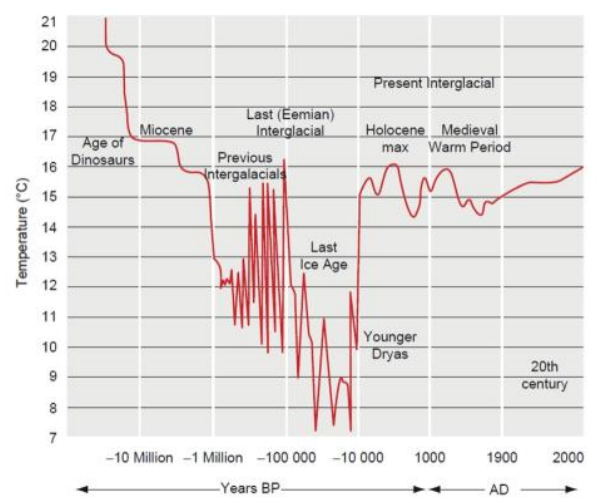

Fig1. Temperature changes over the past 10 million years [5]

In the Pleistocene occurred several ice ages and they totaled six for Europe. During the ice ages, large areas of Northern Europe and many mountain regions of Central and South Eurasia were covered with ice (Fig. 2). In the Last Glacial Maximum (LGM), many parts of Northern and Western Europe located south of the ice sheets and glaciers were covered with permafrost. Southern European peninsulas (such as the Iberian, the Italian and the Balkan) are considered glacial refuges for many species [6]. Between the ice ages occurred periods of warming, which are identified as interglacial periods. These interglacial periods occur over a period of about 100,000 years, and had duration of about 15,000 to 20,000 years. The Last Glacial Maximum occurred before ca. 20,000 years. The transition between the LGM and the current interglacial period has been dramatic. Ice sheets began to retire before ca. 15,000 years as a result of severe sudden warming, called Bolling warm period. This event did not last long and before ca. 12,500 years, the climate suddenly reverted back to glacial temperatures. The last cold snap is called Younger Dryas. The post-glacial warm period began about 11,550 years ago with the glacier retreat from the moraines in Central Scandinavia. With it, Holocene started, the youngest period of the Earth's history [5].

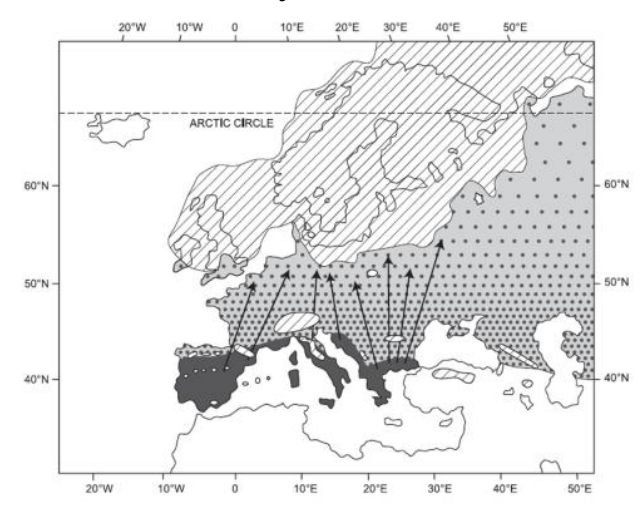

Fig2. Map of Europe in the Pleistocene: the striped areas designate spaces covered with ice; areas in light gray have deep-frozen soil; areas in dark gray are refuges of glacial relicts; the dots on a light gray background show a gradient of refuges of glacial relicts in north-south direction; the arrows show northerner locations of refuges for more species than usually considered [6] 
The aim of our study is to summarize available information about the biodiversity of relict vascular plants that occur on the territory of Bulgaria. Bulgaria is a relatively small country with an area of 110 $994 \mathrm{~km}^{2}$, located on the Balkan Peninsula in Southeastern Europe (Fig. 3). As mentioned above, the Balkan Peninsula was one of the refuges for many species during the glacial periods. At this time, the botanical literature in Bulgaria lacks a publication that contains a list of relict vascular plants and their distribution in different groups. Our goal is to fill this gap. A detailed list of relict plants is needed not only for the purposes of ecology and environmental protection; it will be particularly useful in floristic studies, in conservation biology and in biogeography.

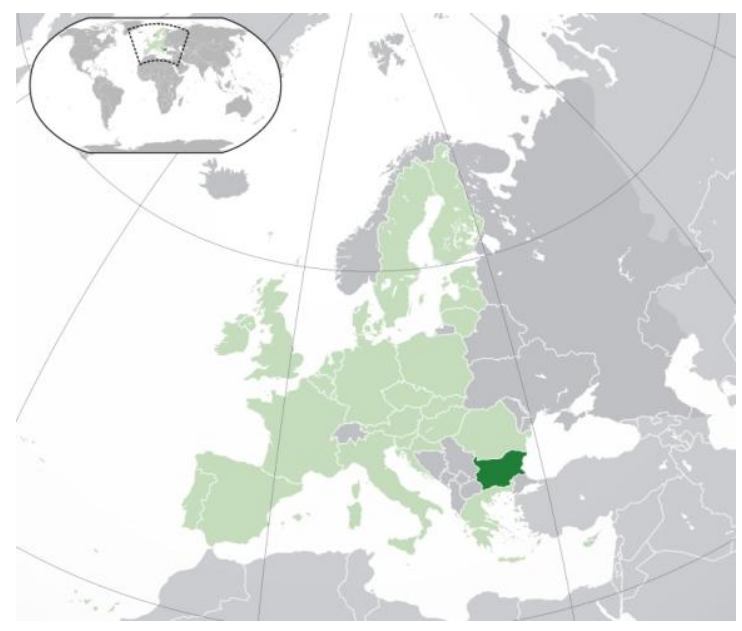

Fig3. Geographical position of Bulgaria

\section{Materials ANd Methods}

Information on individual relict vascular plants can be found in some of the most important botanical publications in Bulgaria: Flora of PR Bulgaria, Vol. 1-7 [7], Flora of PR Bulgaria, Vol. 8-9 [8], Red Data Book of PR Bulgaria, Vol. 1. Plants [9], Biodiversity of Vascular Plants in Bulgaria [10], Red Data Book of Republic of Bulgaria, Vol. 1. Plants and Fungi [11], Red Data Book of Republic of Bulgaria, Vol. 3. Natural Habitats [12], Important Plant Areas in Bulgaria [13] and in plans for management of the protected areas in Bulgaria $[14,15]$. A number of other publications by Bulgarian authors also include data on relict vascular plants in Bulgaria $[16,17,18,19,20,21,22]$. This information may be supplemented by publications by authors from neighboring countries $[23,24,25$, $26,27,28,29,30,31,32,33,34,35,36,37,38,39,40,41,42,43,44,45,46,47,48,49,50]$. The names of the families Angiosperm plants are according Angiosperm Phylogeny Group III [51].

\section{RESUltS AND DisCUSSION}

Based on the information from all sources and our critical selection, we have prepared a list of relict vascular plants that occur on the territory of the Republic of Bulgaria (in Appendix). In this list, we have not included cosmopolitan species that other authors cited as relicts. According to the abovementioned definition of relict species, we are convinced that cosmopolitan species should not be counted in this group.

Relict vascular plants in the Bulgarian flora are 346 species from 207 genera and 81 families. This number accounts for $8.74 \%$ of the natural flora of Bulgaria and $8.43 \%$ of the total flora of Bulgaria (which includes foreign species). We divided relict plants into two groups: tertiary relicts (183 species) and quaternary relicts (163 species). The quaternary relicts are divided into 3 groups: glacial relicts (143 species), interglacial relicts (13 species) and postglacial relicts (7 species).

The families with the highest number of relict species in the Bulgarian flora are: Asteraceae, Fabaceae (19 species), Salicaceae (18 species), Rosaceae (16 species), Cyperaceae, Ericaceae (14 species), Primulaceae (12 species), Campanulaceae, Caryophyllaceae, Saxifragaceae (11 species). The genera with the largest number of relict species are: Salix L. (14 species), Carex L. (10 species), Saxifraga L. (9 species), Acer L., Astragalus L. (7 species), Campanula L., Gentiana L., Pinus L., Quercus L. (6 species).

Among the relict species with the largest number, are perennial herbaceous plants, followed by shrubs and trees (Table 1). 
Table1. Distribution of relict species by biological type

\begin{tabular}{|c|c|c|c|c|}
\hline \multirow{2}{*}{ Biological type } & \multirow{2}{*}{ Tertiary relicts } & \multicolumn{3}{|c|}{ Quaternary relicts } \\
\cline { 3 - 5 } & & Glacial & Interglacial & Postglacial \\
\hline Annual herbaceous plant & 5 & 1 & - & - \\
\hline Annual or biannual herbaceous plant & 1 & 1 & - & - \\
\hline Biannual herbaceous plant & 2 & - & - & - \\
\hline Perennial herbaceous plant & 70 & 123 & 12 & 6 \\
\hline Shrub & 44 & 15 & 1 & 1 \\
\hline Shrub or tree & 23 & 3 & - & - \\
\hline Tree & 38 & - & - & - \\
\hline
\end{tabular}

The distribution of relict species according to their floristic elements, results in the following: Among the tertiary relicts (Fig. 4), most species have sub-Mediterranean origin (22 species), Euro-Asian origin (20 species) and Balkan origin (20 species). Among the quaternary glacial relicts (Fig. 5), approximately $1 / 3$ of the species have Boreal origin (45 species). The Arctic-Alpine species (17 species) and Alpine-Carpathian-Balkan species (14 species) are a relatively large number.

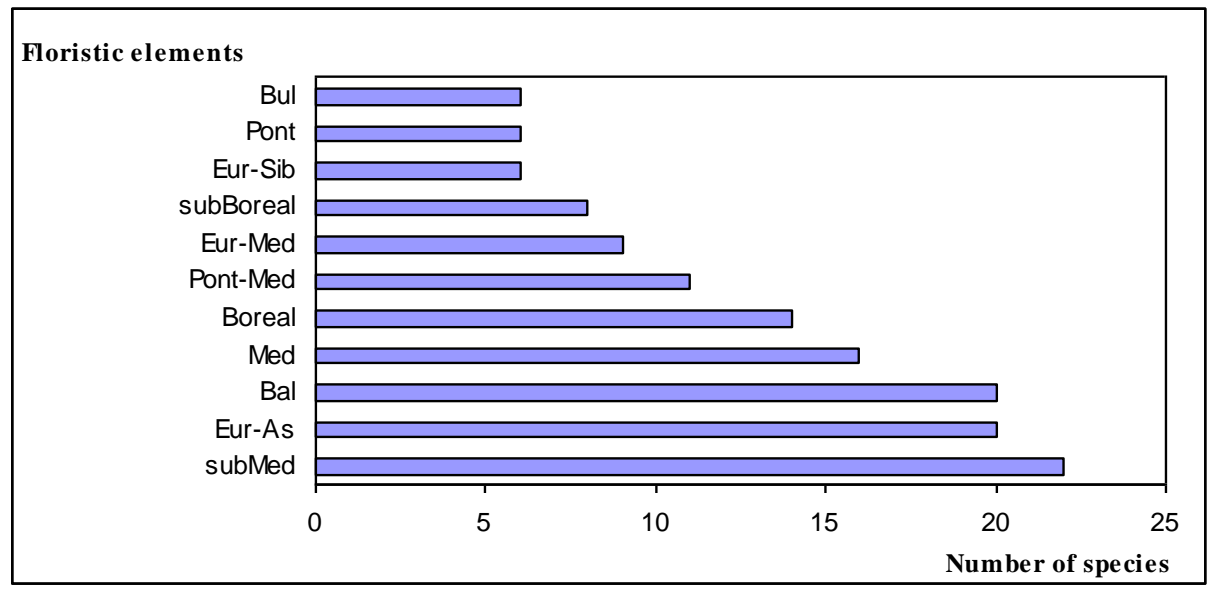

Fig4. Distribution of tertiary relicts by floristic elements

(Only floristic elements represented by 5 or more species are included)

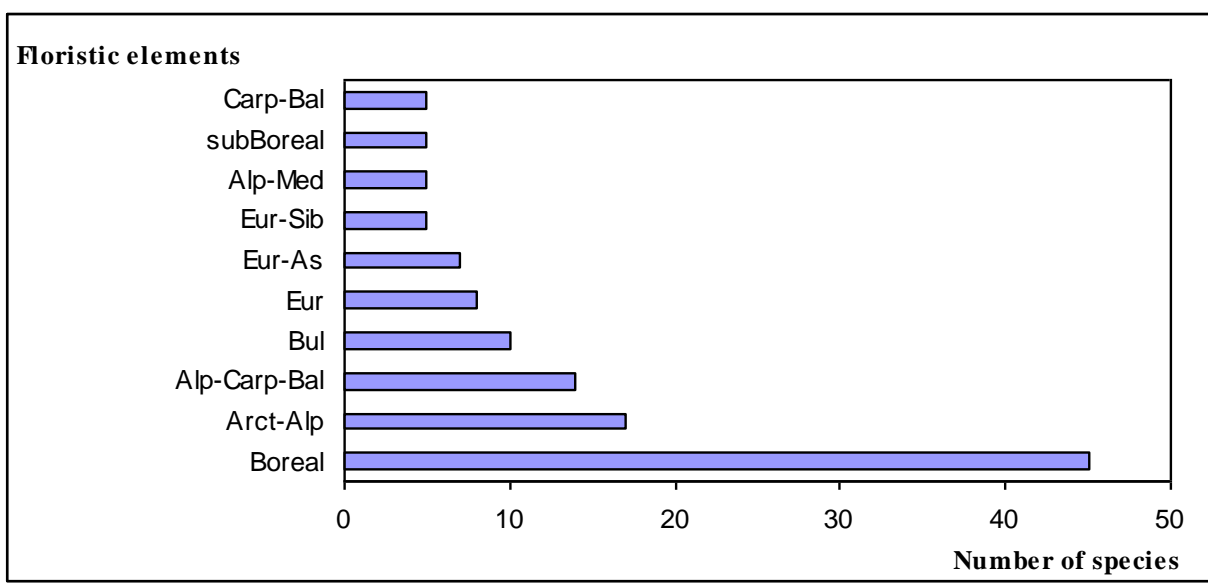

Fig5. Distribution of quaternary glacial relicts by floristic elements

(Only floristic elements represented by 5 or more species are included)

144 relict species have conservation status: 77 species of tertiary relicts, 59 species of quaternary glacial relicts, 6 species of quaternary interglacial relicts and 2 species of postglacial relicts (in Appendix).

\section{Conclusion}

As a result of our study we found a significant number of relict species of vascular plants in the flora of Bulgaria. The list of relicts who we prepared, divided into groups, systematize available data. It will be useful in floristic studies, in conservation biology and in biogeography. 


\section{APPENDIX}

\section{List of Relict Vascular Plants in Bulgaria}

\section{Legend}

\section{- Biological Type}

$\mathrm{a}$ - annual herbaceous plant; $\mathrm{a}-\mathrm{b}$ - annual or biennial herbaceous plant; $\mathrm{b}$ - biennial herbaceous plant;

$\mathrm{p}$ - perennial herbaceous plant; sh - shrub; sh- $\mathrm{t}$ - shrub or tree; $\mathrm{t}$ - tree

\section{- Conservation Status}

Bern Convention [52]: BC1 - Appendix 1; Convention on International Trade in Endangered Species of Wild Fauna and Flora [53]: CITES; Directive 92/43/EEC [54]: DA2 - Annex II, DA4 - Annex IV, DA5 - Annex V; Red Data Book of Republic of Bulgaria. Vol.1. Plants and Fungi [11]: CR Critically endangered, EN - Endangered, EX - Extinct, RE - Regionally extinct, VU - Vulnerable; Biodiversity Act of Republic of Bulgaria [55]: BA2 - Annex 2, BA2a - Annex 2a, BA3 - Annex 3, BA4 - Annex 4.

\section{- Floristic Element}

Alp - Alpine; Anat - Anatolian; Ap - Apenninian; Arct - Arctic; As - Asiatic; Bal - Balkan; Boreal Boreal; Bul - Bulgarian; Carp - Carpathian; Cauc - Caucasus; Dac - Dacian; Eur - European; Eux Euxinian; Med - Mediterranean; OT - Oriental-Turanian; Pont - Pontic; Sib - Siberian; Prefixes: sub - sub; N - North; S - South; E - East; W - West; C - Central.

Table2. Tertiary relicts

\begin{tabular}{|c|c|c|c|c|c|}
\hline No & Species name & Family & $\begin{array}{l}\text { Biological } \\
\text { type }\end{array}$ & $\begin{array}{c}\text { Conser- } \\
\text { vation } \\
\text { status }\end{array}$ & $\begin{array}{l}\text { Floristic } \\
\text { element }\end{array}$ \\
\hline 1 & Abies alba Mill. & Pinaceae & $\mathrm{t}$ & & Boreal \\
\hline 2 & Abies borisii-regis Mattf. & Pinaceae & $\mathrm{t}$ & & Bal \\
\hline 3 & Acer campestre L. & Sapindaceae & sh-t & & Eur-OT \\
\hline 4 & Acer heldreichii Orph. ex Boiss. & Sapindaceae & $\mathrm{t}$ & VU & Bal \\
\hline 5 & Acer hyrcanum Fisch. \& C. A. Mey & Sapindaceae & $\mathrm{t}$ & & subMed \\
\hline 6 & Acer monspessulanum L. & Sapindaceae & $\mathrm{t}$ & & subMed \\
\hline 7 & Acer platanoides L. & Sapindaceae & $\mathrm{t}$ & & subMed \\
\hline 8 & Acer pseudoplatanus L. & Sapindaceae & $\mathrm{t}$ & & Eur-Med \\
\hline 9 & Acer tataricum $\mathrm{L}$. & Sapindaceae & sh-t & & subMed \\
\hline 10 & Adiantum capillus-veneris L. & Adiantaceae & $\mathrm{p}$ & CR, BA3 & subBoreal \\
\hline 11 & Aesculus hippocastanum L. & Sapindaceae & $\mathrm{t}$ & EN, BA3 & Bal \\
\hline 12 & $\begin{array}{l}\text { Aethionema arabicum (L.) Andrz. ex } \\
\text { DC. }\end{array}$ & Brassicaceae & $\mathrm{a}$ & CR, BA3 & subMed \\
\hline 13 & Alnus glutinosa (L.) Gaertn. & Betulaceae & $\mathrm{t}$ & & Med-CAs \\
\hline 14 & Alnus incana (L.) Moench & Betulaceae & $\mathrm{t}$ & & subMed \\
\hline 15 & $\begin{array}{l}\text { Anthemis argyrophylla (Halácsy \& } \\
\text { Georgiev) Velen. }\end{array}$ & Asteraceae & $\mathrm{p}$ & CR, BA3 & Bul \\
\hline 16 & Arbutus andrachne L. & Ericaceae & sh & $\mathrm{CR}, \mathrm{BA} 3$ & Med \\
\hline 17 & Arbutus unedo L. & Ericaceae & sh & $\mathrm{CR}, \mathrm{BA} 3$ & subMed-As \\
\hline 18 & Arctostaphylos uva-ursi (L.) Spreng. & Ericaceae & $\mathrm{sh}$ & & Boreal \\
\hline 19 & Asarum europaeum L. & Aristolochiaceae & $\mathrm{p}$ & & Eur-Sib \\
\hline 20 & Asperula suberosa Sibth. \& Sm. & Rubiaceae & $\mathrm{p}$ & CR, BA3 & Bal \\
\hline 21 & Astracantha aitosensis (Ivan.) Podl. & Fabaceae & $\mathrm{sh}$ & $\begin{array}{l}\text { BC1, EN, } \\
\text { BA3 }\end{array}$ & Bul \\
\hline 22 & $\begin{array}{l}\text { Astracantha thracica (Griseb.) } \\
\text { Podlech }\end{array}$ & Fabaceae & sh & VU, BA3 & Bal \\
\hline 23 & Astragalus physocalyx Fisch. & Fabaceae & sh & $\begin{array}{l}\text { BC1, CR, } \\
\text { BA3 }\end{array}$ & Bal-Anat \\
\hline 24 & Astragalus wilmottianus Stoj. & Fabaceae & $\mathrm{p}$ & EN, BA3 & Bal \\
\hline 25 & Asyneuma limonifolium (L.) Janch. & Campanulaceae & $\mathrm{p}$ & & Ap-Bal \\
\hline 26 & Azolla filiculoides Lam. & Salviniaceae & $\mathrm{a}$ & & Boreal \\
\hline 27 & Berberis vulgaris L. & Berberidaceae & sh & & Eur-Med \\
\hline 28 & Betula pendula Roth & Betulaceae & $\mathrm{t}$ & & Eur-Sib \\
\hline 29 & Blechnum spicant (L.) Sm. & Blechnaceae & $\mathrm{p}$ & & Boreal \\
\hline
\end{tabular}




\begin{tabular}{|c|c|c|c|c|c|}
\hline 30 & Butomus umbellatus L. & Butomaceae & $\mathrm{p}$ & & Eur-As \\
\hline 31 & Caldesia parnassifolia (L.) Parl. & Alismataceae & $\mathrm{p}$ & $\begin{array}{c}\mathrm{BC} 1, \mathrm{DA} 2, \\
\mathrm{RE}, \mathrm{BA} 2, \\
\text { BA3 }\end{array}$ & Eur-As \\
\hline 32 & Calluna vulgaris (L.) Hull & Ericaceae & sh & BA3 & subBoreal \\
\hline 33 & Campanula lanata Friv. & Campanulaceae & $\mathrm{b}$ & $\begin{array}{l}\text { BC1, VU, } \\
\text { BA3 }\end{array}$ & Bal \\
\hline 34 & Campanula lingulata Waldst. \& Kit. & Campanulaceae & $\mathrm{p}$ & & Ap-Bal \\
\hline 35 & Campanula versicolor Andrews & Campanulaceae & $\mathrm{p}$ & EN, BA3 & EMed \\
\hline 36 & Cardamine glauca Spreng. ex DC. & Brassicaceae & $\mathrm{a}$ & & subMed \\
\hline 37 & Cardamine graeca $\mathrm{L}$. & Brassicaceae & $a-b$ & & Med \\
\hline 38 & Carpinus betulus L. & Betulaceae & $\mathrm{t}$ & & Eur-subMed \\
\hline 39 & Carpinus orientalis Mill. & Betulaceae & sh-t & & subMed \\
\hline 40 & Castanea sativa $\mathrm{L}$. & Fagaceae & $\mathrm{t}$ & EN & Med \\
\hline 41 & Celtis australis L. & Ulmaceae & $\mathrm{t}$ & & Med \\
\hline 42 & Celtis plachoniana K.I. Chr. & Ulmaceae & sh-t & EN & Med \\
\hline 43 & Centaurea calocephala Willd. & Asteraceae & $\mathrm{p}$ & $\mathrm{CR}$ & Pont \\
\hline 44 & Centaurea calvescens Pančić & Asteraceae & $\mathrm{b}$ & & subMed \\
\hline 45 & Centaurea jankae D. Brandza & Asteraceae & $\mathrm{p}$ & $\begin{array}{c}\mathrm{BC} 1, \mathrm{DA} 2, \\
\mathrm{EN}, \mathrm{BA} 2, \\
\text { BA3 }\end{array}$ & Bal \\
\hline 46 & Centaurea trinervia Steven ex Willd. & Asteraceae & $\mathrm{p}$ & $\mathrm{CR}$ & Pont \\
\hline 47 & $\begin{array}{l}\text { Centaurea wagenitziana } \text { Bancheva } \\
\& \text { Kit Tan }\end{array}$ & Asteraceae & $\mathrm{p}$ & CR, BA3 & Bal \\
\hline 48 & $\begin{array}{l}\text { Centranthus kellereri (Stoj., Stef. \& } \\
\text { T. Georg.) Stoj. \& Stef. }\end{array}$ & Caprifoliaceae & $\mathrm{p}$ & $\begin{array}{l}\mathrm{BC} 1, \mathrm{CR} \\
\mathrm{BA} 3 \\
\end{array}$ & Bul \\
\hline 49 & Ceratophyllum submersum L. & Ceratophyllaceae & $\mathrm{p}$ & & Eur-As \\
\hline 50 & Cercis siliquastrum $\mathrm{L}$. & Fabaceae & sh-t & & Med-OT \\
\hline 51 & Cicer montbretii Jaub. \& Spach & Fabaceae & $\mathrm{p}$ & EN, BA3 & Bal-Anat \\
\hline 52 & Cistus salviifolius L. & Cistaceae & $\mathrm{sh}$ & EN, BA3 & Eur-As \\
\hline 53 & Clematis vitalba $\mathrm{L}$. & Ranunculaceae & $\mathrm{p}$ & & Eur \\
\hline 54 & Colutea arborescens L. & Fabaceae & $\mathrm{sh}$ & & subMed \\
\hline 55 & $\begin{array}{l}\text { Comandra elegans (Rochel ex } \\
\text { Rchb.) Rchb. f. }\end{array}$ & Santalaceae & sh & & $\begin{array}{l}\text { Bal-Dac- } \\
\text { Anat }\end{array}$ \\
\hline 56 & Corylus avellana L. & Betulaceae & sh & & Med-CAs \\
\hline 57 & Corylus colurna $\mathrm{L}$. & Betulaceae & $\mathrm{t}$ & & Pont-CAs \\
\hline 58 & Cotinus coggygria Scop. & Anacardiaceae & sh & & Med-As \\
\hline 59 & Crataegus pentagyna Waldst. \& Kit. & Rosaceae & sh & & subMed \\
\hline 60 & Cyclamen coum Mill. & Primulaceae & $\mathrm{p}$ & $\begin{array}{c}\text { BC1, } \\
\text { CITES, } \\
\text { BA3 }\end{array}$ & Pont-Med \\
\hline 61 & Cypripedium calceolus L. & Orchidaceae & $\mathrm{p}$ & $\begin{array}{c}\text { BC1, } \\
\text { CITES, } \\
\text { DA2, CR, } \\
\text { BA2, BA3 }\end{array}$ & Eur-As \\
\hline 62 & Daphne kosanini (Stoj.) Stoj. & Thymelaeceae & $\mathrm{sh}$ & & subMed \\
\hline 63 & Daphne laureola L. & Thymelaeceae & sh & EN, BA3 & subMed \\
\hline 64 & Daphne mezereum L. & Thymelaeceae & sh & & Eur-Sib \\
\hline 65 & Daphne pontica L. & Thymelaeceae & sh & EN, BA3 & Pont \\
\hline 66 & Dictamnus albus L. & Rutaceae & $\mathrm{p}$ & & Eur-As \\
\hline 67 & Edraianthus graminifolius (L.) DC. & Campanulaceae & $\mathrm{p}$ & & Bal-Ap \\
\hline 68 & $\begin{array}{l}\text { Edraianthus serbicus (A. Kern.) } \\
\text { Petrovič }\end{array}$ & Campanulaceae & $\mathrm{p}$ & EN, BA3 & Bal \\
\hline 69 & Ephedra distachya $\mathrm{L}$. & Ephedraceae & sh & BA3 & Pont-Med \\
\hline 70 & $\begin{array}{l}\text { Epimedium pubigerum (DC.) Morren } \\
\& \text { Decne }\end{array}$ & Berberidaceae & $\mathrm{p}$ & BA3 & Bal-Anat \\
\hline 71 & Eranthis bulgaricus (Stef.) Stef. & Ranunculaceae & $\mathrm{p}$ & CR, BA3 & Bul \\
\hline 72 & Erica arborea L. & Ericaceae & sh & BA3 & Pont-Med \\
\hline 73 & Erythronium dens-canis L. & Liliaceae & $\mathrm{p}$ & & Med \\
\hline 74 & Fagus orientalis Lipsky & Fagaceae & $\mathrm{t}$ & & SEux \\
\hline 75 & Fraxinus excelsior L. & Oleaceae & $\mathrm{t}$ & & Eur-Med \\
\hline
\end{tabular}


Biodiversity of Relict Vascular Plants in Bulgaria

\begin{tabular}{|c|c|c|c|c|c|}
\hline 76 & Fraxinus ornus L. & Oleaceae & $\mathrm{t}$ & & subMed \\
\hline 77 & Galium procurrens Ehrend. & Rubiaceae & $\mathrm{p}$ & CR, BA3 & Bal \\
\hline 78 & Galium rhodopeum Velen. & Rubiaceae & $\mathrm{p}$ & $\begin{array}{l}\text { BC1, EN, } \\
\text { BA3 }\end{array}$ & Bal \\
\hline 79 & Gentiana frigida Haenke & Gentianaceae & $\mathrm{p}$ & EN, BA3 & Alp-Bal \\
\hline 80 & Gentiana lutea $\mathrm{L}$. & Gentianaceae & $\mathrm{p}$ & $\begin{array}{l}\text { DA5, EN, } \\
\text { BA3 }\end{array}$ & Eur \\
\hline 81 & Goodyera repens (L.) R. Br. & Orchidaceae & $\mathrm{p}$ & $\begin{array}{l}\text { CITES, } \\
\text { EN, BA3 }\end{array}$ & Boreal \\
\hline 82 & Gypsophila tekirae Stef. & Caryophyllaceae & $\mathrm{p}$ & CR, BA3 & Bul \\
\hline 83 & Haberlea rhodopensis Friv. & Gesneriaceae & $\mathrm{p}$ & $\mathrm{BC} 1, \mathrm{BA} 3$ & Bal \\
\hline 84 & Hedera helix L. & Araliaceae & sh & & Eur-As \\
\hline 85 & Herminium monorchis (L.) R. Br. & Orchidaceae & $\mathrm{p}$ & $\begin{array}{c}\text { CITES, RE, } \\
\text { BA3 }\end{array}$ & Eur-As \\
\hline 86 & Hippuris vulgaris L. & Plantaginaceae & $\mathrm{p}$ & $\mathrm{CR}, \mathrm{BA} 3$ & Boreal \\
\hline 87 & Hottonia palustris L. & Primulaceae & $\mathrm{p}$ & RE, BA3 & Boreal \\
\hline 88 & Hydrocharis morsus-ranae L. & Hydrocharitaceae & $\mathrm{p}$ & & Eur-As \\
\hline 89 & Hypericum androsaemum L. & Hypericaceae & sh & EN, BA3 & Eur-OT \\
\hline 90 & Hypericum calycinum L. & Hypericaceae & sh & EN, BA3 & Pont-Med \\
\hline 91 & Hypericum setiferum Stef. & Hypericaceae & $\mathrm{p}$ & EX, BA3 & Bul \\
\hline 92 & Ilex aquifolium $\mathrm{L}$. & Aquifoliaceae & sh-t & EN, BA3 & subMed \\
\hline 93 & Ilex colchica Pojark. & Aquifoliaceae & $\mathrm{sh}$ & EN, BA3 & Pont \\
\hline 94 & Isopyrum thalictroides L. & Ranunculaceae & $\mathrm{p}$ & & Eur \\
\hline 95 & Jasminum fruticans $\mathrm{L}$. & Oleaceae & $\mathrm{sh}$ & & Pont-CAs \\
\hline 96 & Juglans regia L. & Juglandaceae & $\mathrm{t}$ & & Eur-As \\
\hline 97 & Juniperus communis L. & Cupressaceae & sh-t & & subBoreal \\
\hline 98 & Juniperus excelsa M. Bieb. & Cupressaceae & sh-t & BA3 & Med-OT \\
\hline 99 & Juniperus oxycedrus L. & Cupressaceae & sh-t & & Eur-Med \\
\hline 100 & Juniperus sabina L. & Cupressaceae & $\mathrm{sh}$ & EN, BA3 & Pont-Sib \\
\hline 101 & Lathraea rhodopaea Dingler & Scrophulariaceae & $\mathrm{p}$ & BA3 & Bal \\
\hline 102 & Lathyrus aureus (Steven) D. Brândză & Fabaceae & $\mathrm{p}$ & & Eux \\
\hline 103 & Laurocerasus officinalis Roem. & Rosaceae & sh-t & & Eux \\
\hline 104 & Leersia oryzoides (L.) Sw. & Poaceae & $\mathrm{p}$ & & subBoreal \\
\hline 105 & Limodorum abortivum (L.) Sw. & Orchidaceae & $\mathrm{p}$ & $\begin{array}{l}\text { CITES, } \\
\text { BA3 }\end{array}$ & Med \\
\hline 106 & Lonicera etrusca Santi & Caprifoliaceae & sh & & Med \\
\hline 107 & Lycopus europaeus L. & Lamiaceae & $\mathrm{p}$ & & Eur-As \\
\hline 108 & Mespilus germanica L. & Rosaceae & sh-t & & Pont-Med \\
\hline 109 & Micromeria cristata (Hampe) Griseb & Lamiaceae & $\mathrm{p}$ & & Bal-Anat \\
\hline 110 & Oenanthe aquatica (L.) Poir. & Apiaceae & $\mathrm{p}$ & & Eur-Sib \\
\hline 111 & Ostrya carpinifolia Scop. & Betulaceae & sh-t & & subMed \\
\hline 112 & Paeonia mascula (L.) Mill. & Paeoniaceae & $\mathrm{p}$ & EN, BA3 & Pont-Med \\
\hline 113 & Paliurus spina-christi Mill. & Rhamnaceae & sh & & Eur-As \\
\hline 114 & Pancratium maritimum L. & Amaryllidaceae & $\mathrm{p}$ & EN, BA3 & Pont-Med \\
\hline 115 & Periploca graeca $\mathrm{L}$. & Apocynaceae & $\mathrm{sh}$ & & Pont-Med \\
\hline 116 & Phillyrea latifolia L. & Oleaceae & sh-t & & Med \\
\hline 117 & Picea abies (L.) Karst. & Pinaceae & $\mathrm{t}$ & & Boreal \\
\hline 118 & Pinus brutia Ten. & Pinaceae & $\mathrm{t}$ & CR, BA3 & Med \\
\hline 119 & Pinus heldreichii Christ. & Pinaceae & $\mathrm{t}$ & & Ap-Bal \\
\hline 120 & Pinus nigra J.F. Arnold & Pinaceae & $\mathrm{t}$ & & subMed \\
\hline 121 & Pinus peuce Griseb. & Pinaceae & $\mathrm{t}$ & & Bal \\
\hline 122 & Pinus sylvestris L. & Pinaceae & $\mathrm{t}$ & & subBoreal \\
\hline 123 & Pistacia terebinthus L. & Anacardiaceae & sh-t & & Pont-Med \\
\hline 124 & Platanus orientalis L. & Platanaceae & $\mathrm{t}$ & & Med \\
\hline 125 & Populus alba L. & Salicaceae & $\mathrm{t}$ & & Eur-As \\
\hline 126 & Populus canescens (Aiton) Sm. & Salicaceae & $\mathrm{t}$ & & Eur-Med \\
\hline 127 & Populus nigra L. & Salicaceae & $\mathrm{t}$ & & Eur-As \\
\hline 128 & Populus tremula L. & Salicaceae & $\mathrm{t}$ & & subBoreal \\
\hline 129 & Potentilla palustris (L.) Scop. & Rosaceae & sh & BA3 & Boreal \\
\hline 130 & $\begin{array}{l}\text { Primula vulgaris subsp. rubra (Sm.) } \\
\text { Arcang. }\end{array}$ & Primulaceae & $\mathrm{p}$ & BA4 & Bal-Eux \\
\hline
\end{tabular}




\begin{tabular}{|c|c|c|c|c|c|}
\hline 131 & Prunus fruticosa Pall. & Rosaceae & sh & & Eur-Sib \\
\hline 132 & Pyracantha coccinea M.J. Roemer & Rosaceae & sh & & Pont-Med \\
\hline 133 & Quercus cerris L. & Fagaceae & $\mathrm{t}$ & & Eur-subMed \\
\hline 134 & Quercus coccifera L. & Fagaceae & sh-t & EN, BA3 & Med \\
\hline 135 & Quercus dalechampii Ten. & Fagaceae & $\mathrm{t}$ & & subMed \\
\hline 136 & Quercus hartwissiana Steven & Fagaceae & $\mathrm{t}$ & BA2a & Pont-Eux \\
\hline 137 & Quercus pubescens Willd. & Fagaceae & sh-t & & Eur-subMed \\
\hline 138 & Quercus trojana Webb & Fagaceae & $\mathrm{t}$ & BA3 & Ap-Bal-Anat \\
\hline 139 & Ramonda serbica Pančič & Gesneriaceae & $\mathrm{p}$ & $\begin{array}{c}\text { BC1, DA4, } \\
\text { EN, BA3 }\end{array}$ & Bal \\
\hline 140 & Ranunculus lingua $\mathrm{L}$. & Ranunculaceae & $\mathrm{p}$ & & Eur-As \\
\hline 141 & Rhododendron ponticum L. & Ericaceae & sh & BA3 & Eux \\
\hline 142 & Rhus coriaria $\mathrm{L}$. & Anacardiaceae & sh-t & & Med-As \\
\hline 143 & Rhynchocorys elephas (L.) Griseb. & Orobanchaceae & $\mathrm{p}$ & BA3 & subMed \\
\hline 144 & Rumex acetosa $\mathrm{L}$. & Polygonaceae & $\mathrm{p}$ & & Boreal \\
\hline 145 & Ruscus aculeatus L. & Asparagaceae & $\mathrm{p}$ & DA5, BA4 & SPont \\
\hline 146 & Ruscus hypoglossum L. & Asparagaceae & $\mathrm{p}$ & BA4 & Pont \\
\hline 147 & Ruta graveolens L. & Rutaceae & $\mathrm{p}$ & EN, BA3 & Pont-Med \\
\hline 148 & Salix alba L. & Salicaceae & $\mathrm{t}$ & & Eur-As \\
\hline 149 & Salix caprea L. & Salicaceae & sh-t & BA4 & subBoreal \\
\hline 150 & Salix cinerea $\mathrm{L}$. & Salicaceae & sh & & Eur-As \\
\hline 151 & Salix fragilis L. & Salicaceae & $\mathrm{t}$ & & Eur-As \\
\hline 152 & Salix purpurea $\mathrm{L}$. & Salicaceae & $\mathrm{sh}$ & & $\begin{array}{l}\text { Eur-Med- } \\
\text { CAs }\end{array}$ \\
\hline 153 & Salix silesiaca Willd. & Salicaceae & sh-t & & Carp-Bal \\
\hline 154 & Salix triandra $\mathrm{L}$. & Salicaceae & sh-t & & subBoreal \\
\hline 155 & Salvinia natans (L.) All. & Salviniaceae & $\mathrm{a}$ & $\mathrm{BC} 1, \mathrm{BA} 3$ & Boreal \\
\hline 156 & Sambucus racemosa L. & Caprifoliaceae & sh & & Boreal \\
\hline 157 & Sanicula europaea L. & Apiaceae & $\mathrm{p}$ & & Eur-Sib \\
\hline 158 & Scorzonera lanata M. Bieb. & Asteraceae & $\mathrm{p}$ & & Med \\
\hline 159 & Serapias vomeracea (Burm.) Briq. & Orchidaceae & $\mathrm{p}$ & $\begin{array}{c}\text { CITES, } \\
\text { EN, BA3 }\end{array}$ & Med \\
\hline 160 & Silene asterias Griseb. & Caryophyllaceae & $\mathrm{p}$ & & Bal \\
\hline 161 & Silene lerchenfeldiana Baumg. & Caryophyllaceae & $\mathrm{p}$ & & Carp-Bal \\
\hline 162 & Silene waldsteinii Griseb. & Caryophyllaceae & $\mathrm{p}$ & & $\mathrm{Bal}$ \\
\hline 163 & Smilax excelsa $\mathrm{L}$ & Smilacaceae & sh & & subMed \\
\hline 164 & Sorbus aria (L.) Crantz & Rosaceae & sh-t & & Eur \\
\hline 165 & Staphylea pinnata $\mathrm{L}$. & Staphyleaceae & sh-t & & Eur-Med \\
\hline 166 & Stratiotes aloides L. & Hydrocharitaceae & $\mathrm{p}$ & CR, BA3 & Eur-Sib \\
\hline 167 & Syringa vulgaris L. & Oleaceae & sh & & Carp-Bal \\
\hline 168 & Tamus communis L. & Dioscoreaceae & $\mathrm{p}$ & & subMed \\
\hline 169 & Taraxacum bithynicum DC. & Asteraceae & $\mathrm{p}$ & EN & Med \\
\hline 170 & Taxus baccata L. & Taxaceae & sh-t & EN, BA3 & Eur-NAm \\
\hline 171 & Trachelium rumelianum Hampe & Campanulaceae & $\mathrm{p}$ & VU, BA3 & Bal \\
\hline 172 & Trachystemon orientalis (L.) D. Don & Boraginaceae & $\mathrm{p}$ & & SEux \\
\hline 173 & Trapa natans $\mathrm{L}$. & Lythraceae & $\mathrm{p}$ & $\begin{array}{c}\text { BC1, EN, } \\
\text { BA3 }\end{array}$ & Eur-As \\
\hline 174 & $\begin{array}{l}\text { Turgeniopsis foeniculacea (Fenzl) } \\
\text { Boiss. }\end{array}$ & Apiaceae & $\mathrm{a}$ & CR, BA3 & Med \\
\hline 175 & Ulmus laevis Pall. & Ulmaceae & $\mathrm{t}$ & & Eur-Med \\
\hline 176 & Ulmus minor Mill. & Ulmaceae & $\mathrm{t}$ & & Eur-Med \\
\hline 177 & Vaccinium arctostaphylos L. & Ericaceae & sh & $\begin{array}{l}\mathrm{BC} 1, \mathrm{EN}, \\
\mathrm{BA} 3\end{array}$ & Pont \\
\hline 178 & Vaccinium myrtillus L. & Ericaceae & sh & & Boreal \\
\hline 179 & Vaccinium uliginosum $\mathrm{L}$. & Ericaceae & sh & & Boreal \\
\hline 180 & Veronica turrilliana Stoj. \& Stef. & Scrophulariaceae & $\mathrm{p}$ & $\begin{array}{c}\text { BC1, EN, } \\
\text { BA3 }\end{array}$ & Bal \\
\hline 181 & Viburnum lantana L. & Caprifoliaceae & sh & & Eur-Med \\
\hline 182 & Viscum album L. & Loranthaceae & sh & & Eur-As \\
\hline 183 & Vitis sylvestris C. C. Gmel. & Vitaceae & sh & & subMed \\
\hline
\end{tabular}


Table3. Quaternary glacial relicts

\begin{tabular}{|c|c|c|c|c|c|}
\hline No & Species name & Family & $\begin{array}{c}\text { Biological } \\
\text { type }\end{array}$ & $\begin{array}{c}\text { Conser-vation } \\
\text { status } \\
\end{array}$ & $\begin{array}{l}\text { Floristic } \\
\text { element }\end{array}$ \\
\hline 1 & $\begin{array}{l}\text { Adenostyles alliariae (Gouan) A. } \\
\text { Kern. }\end{array}$ & Asteraceae & $\mathrm{p}$ & & Alp-Carp-Bal \\
\hline 2 & $\begin{array}{l}\text { Alyssum orbelicum Ančev \& } \\
\text { Uzunov }\end{array}$ & Brassicaceae & $\mathrm{p}$ & CR, BA3 & Bul \\
\hline 3 & $\begin{array}{l}\text { Alyssum pirinicum (Stoj. \& Acht.) } \\
\text { Ančev }\end{array}$ & Brassicaceae & $\mathrm{p}$ & EN, BA3 & Bul \\
\hline 4 & Androsace villosa $\mathrm{L}$ & Primulaceae & $\mathrm{p}$ & & Arct-Alp \\
\hline 5 & Anemone narcissiflora L. & Ranunculaceae & $\mathrm{p}$ & BA3 & Boreal \\
\hline 6 & Antennaria dioica (L.) Gaerth. & Asteraceae & $\mathrm{p}$ & & Arct-Alp \\
\hline 7 & Arabis alpina $\mathrm{L}$. & Brassicaceae & $\mathrm{p}$ & & Arct-Alp \\
\hline 8 & $\begin{array}{l}\text { Arabis ferdinandi-coburgii Kellerer } \\
\text { \& Sünd. }\end{array}$ & Brassicaceae & $\mathrm{p}$ & EN, BA3 & Bul \\
\hline 9 & Arenaria biflora $\mathrm{L}$. & Caryophyllaceae & $\mathrm{p}$ & & Alp-Carp-Bal \\
\hline 10 & Arenaria ciliata $\mathrm{L}$. & Caryophyllaceae & $\mathrm{p}$ & EN, BA3 & Alp \\
\hline 11 & Arenaria pirinica Stoj. & Caryophyllaceae & $\mathrm{p}$ & EN, BA3 & Bul \\
\hline 12 & Armeria alpina (DC.) Willd. & Plumbaginaceae & $\mathrm{p}$ & & Alp-Carp-Bal \\
\hline 13 & Artemisia chamaemelifolia Vill. & Asteraceae & sh & CR, BA3 & Alp-Cauc \\
\hline 14 & Artemisia eriantha Ten. & Asteraceae & $\mathrm{p}$ & DA5, EN, BA3 & Carp-Bal \\
\hline 15 & Aster alpinus L. & Asteraceae & $\mathrm{p}$ & & Arct-Alp \\
\hline 16 & Astragalus alopecurus Pall. & Fabaceae & $\mathrm{p}$ & $\begin{array}{l}\text { BC1, DA2, } \\
\text { CR, BA3 }\end{array}$ & Alp \\
\hline 17 & Astragalus australis (L.) Lam. & Fabaceae & $\mathrm{p}$ & & Eur-As \\
\hline 18 & Bartsia alpina $\mathrm{L}$. & Orobanchaceae & $\mathrm{p}$ & & Boreal \\
\hline 19 & Bistorta major S. Gray & Polygonaceae & $\mathrm{p}$ & & Eur-As \\
\hline 20 & Bistorta vivipara (L.) S. Gray & Polygonaceae & $\mathrm{p}$ & & Boreal \\
\hline 21 & Campanula alpina Jacq. & Campanulaceae & $\mathrm{p}$ & & Alp-Carp \\
\hline 22 & Campanula cochlearifolia Lam. & Campanulaceae & $\mathrm{p}$ & EN & Alp-Carp \\
\hline 23 & Campanula rotundifolia $\mathrm{L}$. & Campanulaceae & $\mathrm{p}$ & & Boreal \\
\hline 24 & Carex appropinquata A. Schumach. & Cyperaceae & $\mathrm{p}$ & & Eur-Sib \\
\hline 25 & Carex atrata $\mathrm{L}$. & Cyperaceae & $\mathrm{p}$ & & Eur \\
\hline 26 & Carex ericetorum Pollich & Cyperaceae & $\mathrm{p}$ & & Eur-Sib \\
\hline 27 & Carex curvula All. & Cyperaceae & $\mathrm{p}$ & & Alp-Med \\
\hline 28 & Carex flava $\mathrm{L}$. & Cyperaceae & $\mathrm{p}$ & & Boreal \\
\hline 29 & Carex limosa L. & Cyperaceae & $\mathrm{p}$ & EN, BA2a & Boreal \\
\hline 30 & Carex parviflora Host & Cyperaceae & $\mathrm{p}$ & & Alp \\
\hline 31 & Carex pirinensis Acht. & Cyperaceae & $\mathrm{p}$ & BA2a & Bul \\
\hline 32 & Carex rostrata Stockes & Cyperaceae & $\mathrm{p}$ & & Boreal \\
\hline 33 & Carex rupestris All. & Cyperaceae & $\mathrm{p}$ & BA2a & Boreal \\
\hline 34 & Cerastium alpinum $\mathrm{L}$. & Caryophyllaceae & $\mathrm{p}$ & & Arct-Alp \\
\hline 35 & Cortusa matthioli $\mathrm{L}$. & Primulaceae & $\mathrm{p}$ & BA3 & Arct-Alp \\
\hline 36 & Diphasiastrum alpinum (L.) Holub. & Lycopodiaceae & $\mathrm{p}$ & DA5, BA3 & Boreal \\
\hline 37 & Draba siliquosa M. Bieb. & Brassicaceae & $\mathrm{p}$ & EN & Alp-Carp \\
\hline 38 & Drosera rotundifolia L. & Droseraceae & $\mathrm{p}$ & BA3 & Boreal \\
\hline 39 & Dryas octopetala L. & Rosaceae & sh & & Boreal \\
\hline 40 & $\begin{array}{l}\text { Empetrum nigrum subsp. } \\
\text { hermaphroditum (Hagerup) Böcher. }\end{array}$ & Ericaceae & sh & EN, BA3 & Boreal \\
\hline 41 & Epilobium alsinifolium Vill. & Onagraceae & $\mathrm{p}$ & & Eur \\
\hline 42 & Epilobium anagallidifolium Lam. & Onagraceae & $\mathrm{p}$ & & Boreal \\
\hline 43 & Epilobium palustre L. & Onagraceae & $\mathrm{p}$ & & subBoreal \\
\hline 44 & Equisetum fluviatile L. & Equisetaceae & $\mathrm{p}$ & & Boreal \\
\hline 45 & Eriophorum gracile Koch ex Roth & Cyperaceae & $\mathrm{p}$ & & Boreal \\
\hline 46 & Eriophorum vaginatum L. & Cyperaceae & $\mathrm{p}$ & & Eur-As \\
\hline 47 & Festuca violacea Ser. ex Gaudin & Poaceae & $\mathrm{p}$ & & Boreal \\
\hline 48 & Galanthus nivalis L. & Amaryllidaceae & $\mathrm{p}$ & $\begin{array}{l}\text { CITES, DA5, } \\
\text { EN, BA3 }\end{array}$ & Eur \\
\hline 49 & Galium boreale L. & Rubiaceae & $\mathrm{p}$ & & Boreal \\
\hline 50 & Gentiana acaulis $\mathrm{L}$. & Gentianaceae & $\mathrm{p}$ & CR, BA3 & Eur \\
\hline
\end{tabular}




\begin{tabular}{|c|c|c|c|c|c|}
\hline 51 & Gentiana asclepiadea L. & Gentianaceae & $\mathrm{p}$ & & Eur \\
\hline 52 & Gentiana nivalis L. & Gentianaceae & $\mathrm{a}$ & EN & Boreal \\
\hline 53 & Gentiana verna $\mathrm{L}$. & Gentianaceae & $\mathrm{p}$ & & Eur-As \\
\hline 54 & $\begin{array}{l}\text { Gentianella bulgarica (Velen.) } \\
\text { Holub }\end{array}$ & Gentianaceae & $a-b$ & & Pont \\
\hline 55 & Geum montanum L. & Rosaceae & $\mathrm{p}$ & & Alp-Carp-Bal \\
\hline 56 & Geum reptans $\mathrm{L}$. & Rosaceae & $\mathrm{p}$ & & Alp-Carp-Bal \\
\hline 57 & Gymnadenia conopsea (L.) R. Br. & Orchidaceae & $\mathrm{p}$ & CITES & Eur-As \\
\hline 58 & Hieracium villosum Jacq. & Asteraceae & $\mathrm{p}$ & EN & Alp-Ap-Bal \\
\hline 59 & Homogyne alpina (L.) Cass. & Asteraceae & $\mathrm{p}$ & & Alp-Carp-Bal \\
\hline 60 & Isoëtes lacustris L. & Isoetaceae & $\mathrm{p}$ & EN, BA3 & Boreal \\
\hline 61 & Juncus alpinus Vill. & Juncaceae & $\mathrm{p}$ & & Eur-NAm \\
\hline 62 & Juncus trifidus L. & Juncaceae & $\mathrm{p}$ & & Boreal \\
\hline 63 & Juncus triglumis L. & Juncaceae & $\mathrm{p}$ & CR, BA3 & Arct-Alp \\
\hline 64 & Juniperus sibirica Burgsd. & Cupressaceae & $\operatorname{sh}$ & & Boreal \\
\hline 65 & Kobresia myosuroides (Vill.) Fiori & Cyperaceae & $\mathrm{p}$ & EN, BA2a & Arct-Alp \\
\hline 66 & Laserpitium archangelica Wulfen & Apiaceae & $\mathrm{p}$ & CR, BA3 & Carp-Bal \\
\hline 67 & Ligularia sibirica (L.) Cass. & Asteraceae & $\mathrm{p}$ & $\begin{array}{c}\text { BC1, DA2, } \\
\text { CR, BA2, BA3 }\end{array}$ & Eur-Sib \\
\hline 68 & Listera cordata (L.) R. Br. & Orchidaceae & $\mathrm{p}$ & CITES, BA3 & Boreal \\
\hline 69 & Lloydia serotina (L.) Rchb. & Liliaceae & $\mathrm{p}$ & EN, BA3 & Boreal \\
\hline 70 & Luzula italica Parl. & Juncaceae & $\mathrm{p}$ & & subBoreal \\
\hline 71 & Lysimachia thyrsiflora $\mathrm{L}$. & Primulaceae & $\mathrm{p}$ & BA3 & subBoreal \\
\hline 72 & Menyanthes trifoliata L. & Menyanthaceae & $\mathrm{p}$ & EN, BA3 & Boreal \\
\hline 73 & Meum athamanticum Jacq. & Apiaceae & $\mathrm{p}$ & & Alp-Carp-Bal \\
\hline 74 & Minuartia verna (L.) Hiern & Caryophyllaceae & $\mathrm{p}$ & & subBoreal \\
\hline 75 & Myricaria germanica (L.) Desv. & Tamaricaceae & sh & $\mathrm{CR}, \mathrm{BA} 3$ & Eur-WAs \\
\hline 76 & Nigritella nigra (L.) Rchb. f. & Orchidaceae & $\mathrm{p}$ & CITES & Eur \\
\hline 77 & $\begin{array}{l}\text { Omalotheca norvegica (Gunn.) } \\
\text { Schultz-Bip. \& F. Schultz }\end{array}$ & Asteraceae & $\mathrm{p}$ & & Boreal \\
\hline 78 & Omalotheca supina (L.) DC. & Asteraceae & $\mathrm{p}$ & & Boreal \\
\hline 79 & Oxyria digyna (L.) Hill. & Polygonaceae & $\mathrm{p}$ & & Arct-Alp \\
\hline 80 & Oxytropis campestris (L.) DC. & Fabaceae & $\mathrm{p}$ & & Boreal \\
\hline 81 & $\begin{array}{l}\text { Oxytropis kozhuharovii D. Pavlova, } \\
\text { D. Dimitrov \& M. Nikolova }\end{array}$ & Fabaceae & $\mathrm{p}$ & CR, BA2a & Bul \\
\hline 82 & Oxytropis urumovii Jav. & Fabaceae & $\mathrm{p}$ & VU, BA2a & Bul \\
\hline 83 & $\begin{array}{l}\text { Papaver degenii (Urum. \& Jav.) } \\
\text { Kuzmanov }\end{array}$ & Papaveraceae & $\mathrm{p}$ & VU, BA3 & Bul \\
\hline 84 & Parnassia palustris L. & Saxifragaceae & $\mathrm{p}$ & & subBoreal \\
\hline 85 & Pedicularis oederi Vahl & Scrophulariaceae & $\mathrm{p}$ & EN & Arct-Alp \\
\hline 86 & Pedicularis orthantha Griseb. & Scrophulariaceae & $\mathrm{p}$ & & Bal \\
\hline 87 & Pedicularis verticillata $\mathrm{L}$. & Scrophulariaceae & $\mathrm{p}$ & & Boreal \\
\hline 88 & Phleum alpinum L. & Poaceae & $\mathrm{p}$ & & Arct-Alp \\
\hline 89 & Phyteuma confusum A. Kern. & Campanulaceae & $\mathrm{p}$ & & Alp-Carp-Bal \\
\hline 90 & Pinus mugo Turra & Pinaceae & sh-t & & Alp-Carp-Bal \\
\hline 91 & $\begin{array}{l}\text { Pleuropteropyrum undulatum (Raf.) } \\
\text { Á. Löve \& D. Löve }\end{array}$ & Polygonaceae & $\mathrm{p}$ & & Eur-As \\
\hline 92 & Poa alpina $\mathrm{L}$. & Poaceae & $\mathrm{p}$ & & Boreal \\
\hline 93 & Poa laxa Haenke & Poaceae & $\mathrm{p}$ & & Alp-Med \\
\hline 94 & Poa media Schur & Poaceae & $\mathrm{p}$ & & Carp-Bal \\
\hline 95 & Polygonum viviparum L. & Polygonaceae & $\mathrm{p}$ & & Boreal \\
\hline 96 & Potamogeton alpinus Balb. & Potamogetonaceae & $\mathrm{p}$ & & Boreal \\
\hline 97 & $\begin{array}{l}\text { Potentilla crantzii (Crantz) Beck ex } \\
\text { Fritsch }\end{array}$ & Rosaceae & $\mathrm{p}$ & & Arct-Alp \\
\hline 98 & Potentilla fruticosa $\mathrm{L}$. & Rosaceae & sh & BA3 & Boreal \\
\hline 99 & Primula deorum Velen. & Primulaceae & $\mathrm{p}$ & BC1, VU, BA3 & Bul \\
\hline 100 & Primula farinosa $\mathrm{L}$. & Primulaceae & $\mathrm{p}$ & & Eur \\
\hline 101 & Primula halleri J. F. Gmel. & Primulaceae & $\mathrm{p}$ & BA3 & Alp-Carp-Bal \\
\hline 102 & Primula minima $\mathrm{L}$. & Primulaceae & $\mathrm{p}$ & & Alp-Carp-Bal \\
\hline 103 & $\begin{array}{l}\text { Pseudorchis albida (L.) A. Löve \& } \\
\text { D. Löve }\end{array}$ & Orchidaceae & $\mathrm{p}$ & CITES & Arct-Alp \\
\hline
\end{tabular}




\begin{tabular}{|c|c|c|c|c|c|}
\hline 104 & Pyrola chlorantha $\mathrm{Sw}$. & Ericaceae & $\mathrm{p}$ & & Boreal \\
\hline 105 & Ranunculus crenatus Waldst. \& Kit. & Ranunculaceae & $\mathrm{p}$ & & Alp-Carp-Bal \\
\hline 106 & Rheum rhaponticum $\mathrm{L}$. & Polygonaceae & $\mathrm{p}$ & $\mathrm{BC} 1, \mathrm{CR}, \mathrm{BA} 3$ & Bul \\
\hline 107 & Rhodiola rosea $\mathrm{L}$. & Crassulaceae & $\mathrm{p}$ & CR, BA3 & Boreal \\
\hline 108 & $\begin{array}{l}\text { Rhododendron myrtifolium Schott \& } \\
\text { Kotschy }\end{array}$ & Ericaceae & sh & EN, BA3 & Carp-Bal \\
\hline 109 & Ribes nigrum $\mathrm{L}$. & Saxifragaceae & sh & CR, BA3 & Arct-Alp \\
\hline 110 & Sagina saginoides (L.) Karst. & Caryophyllaceae & $\mathrm{p}$ & & Boreal \\
\hline 111 & Salix appendiculata $\mathrm{L}$. & Salicaceae & sh-t & & Alp-Bal \\
\hline 112 & Salix herbacea L. & Salicaceae & $\mathrm{sh}$ & & Boreal \\
\hline 113 & Salix lapponum L. & Salicaceae & sh & & Eur-Sib \\
\hline 114 & Salix pentandra L. & Salicaceae & sh-t & CR, BA3 & Eur-Sib \\
\hline 115 & Salix reticulata $\mathrm{L}$. & Salicaceae & $\mathrm{sh}$ & & Arct-Alp \\
\hline 116 & Salix retusa $\mathrm{L}$. & Salicaceae & sh & EN, BA3 & Alp \\
\hline 117 & Salix waldsteiniana Willd. & Salicaceae & $\mathrm{sh}$ & & Alp-Carp-Bal \\
\hline 118 & Saxifraga aizoides $\mathrm{L}$. & Saxifragaceae & $\mathrm{p}$ & EN, BA3 & Boreal \\
\hline 119 & Saxifraga androsacea L. & Saxifragaceae & $\mathrm{p}$ & BA3 & Eur-Sib \\
\hline 120 & Saxifraga bryoides L. & Saxifragaceae & $\mathrm{p}$ & & Alp-Med \\
\hline 121 & Saxifraga carpatica Sternb. & Saxifragaceae & $\mathrm{p}$ & & Carp-Bal \\
\hline 122 & $\begin{array}{l}\text { Saxifraga ferdinandi-coburgi } \\
\text { Kellerer \& Sünd. }\end{array}$ & Saxifragaceae & $\mathrm{p}$ & & Bal \\
\hline 123 & Saxifraga oppositifolia L. & Saxifragaceae & $\mathrm{p}$ & & Arct-Alp \\
\hline 124 & Saxifraga paniculata Mill. & Saxifragaceae & $\mathrm{p}$ & & Eur-Am \\
\hline 125 & Saxifraga retusa Gouan & Saxifragaceae & $\mathrm{p}$ & BA3 & $\begin{array}{l}\text { Alp-Ap-Carp- } \\
\text { Bal }\end{array}$ \\
\hline 126 & Saxifraga stellaris L. & Saxifragaceae & $\mathrm{p}$ & & Boreal \\
\hline 127 & $\begin{array}{l}\text { Selaginella selaginoides (L.) P. } \\
\text { Beauv. ex Mart. \& Schrank }\end{array}$ & Selaginellaceae & $\mathrm{p}$ & & Boreal \\
\hline 128 & Sibbaldia procumbens L. & Rosaceae & $\mathrm{p}$ & EN & Boreal \\
\hline 129 & Silene acaulis $\mathrm{L}$. & Caryophyllaceae & $\mathrm{p}$ & & Arct-Alp \\
\hline 130 & Soldanella pusilla Baumg. & Primulaceae & $\mathrm{p}$ & & Alp-Carp-Bal \\
\hline 131 & Soldanella rhodopaea F. K. Mey & Primulaceae & $\mathrm{p}$ & & Bal \\
\hline 132 & Spiraea salicifolia L. & Rosaceae & $\mathrm{sh}$ & CR, BA3 & Boreal \\
\hline 133 & Swertia perennis L. & Gentianaceae & $\mathrm{p}$ & EN & Eur \\
\hline 134 & Swertia punctata Baumg. & Gentianaceae & $\mathrm{p}$ & CR, BA3 & $\begin{array}{l}\text { Carp-Bal- } \\
\text { Cauc }\end{array}$ \\
\hline 135 & Tozzia carpatica Woloszack & Scrophulariaceae & $\mathrm{p}$ & $\begin{array}{l}\text { DA2, VU, } \\
\text { BA2, BA3 }\end{array}$ & Alp-Med \\
\hline 136 & $\begin{array}{l}\text { Trichophorum caespitosum (L.) } \\
\text { Hartm. }\end{array}$ & Cyperaceae & $\mathrm{p}$ & & Boreal \\
\hline 137 & Trollius europaeus L. & Ranunculaceae & $\mathrm{p}$ & BA3 & Boreal \\
\hline 138 & Vaccinium vitis-idaea $\mathrm{L}$. & Ericaceae & $\mathrm{sh}$ & & Boreal \\
\hline 139 & Veratrum lobelianum Bernh. & Melanthiaceae & $\mathrm{p}$ & & Eur-As \\
\hline 140 & Veronica alpina $\mathrm{L}$. & Scrophulariaceae & $\mathrm{p}$ & & Eur-Jap-Ch \\
\hline 141 & Veronica bellidioides $\mathrm{L}$. & Scrophulariaceae & $\mathrm{p}$ & & Alp-Med \\
\hline 142 & Viola biflora $\mathrm{L}$. & Violaceae & $\mathrm{p}$ & & Boreal \\
\hline 143 & Viola palustris L. & Violaceae & $\mathrm{p}$ & $\mathrm{CR}, \mathrm{BA} 3$ & Eur-NAm \\
\hline
\end{tabular}

Table4. Quaternary interglacial relicts

\begin{tabular}{|c|l|c|c|c|c|}
\hline No & \multicolumn{1}{|c|}{ Species name } & Family & $\begin{array}{c}\text { Biological } \\
\text { type }\end{array}$ & $\begin{array}{c}\text { Conser-vation } \\
\text { status }\end{array}$ & $\begin{array}{c}\text { Floristic } \\
\text { element }\end{array}$ \\
\hline 1 & Adonis vernalis L. & Ranunculaceae & $\mathrm{p}$ & CITES & Eur-Sib \\
\hline 2 & Amygdalus nana L. & Rosaceae & $\mathrm{sh}$ & & Eur-As \\
\hline 3 & Asparagus tenuifolius Lam. & Asparagaceae & $\mathrm{p}$ & $\mathrm{BA} 4$ & Pont-Med \\
\hline 4 & Aster oleifolius (Lam.) Wagenitz & Asteraceae & $\mathrm{p}$ & & Pont-Sib \\
\hline 5 & Astragalus austriacus L. & Fabaceae & $\mathrm{p}$ & & subMed \\
\hline 6 & Astragalus pubiflorus DC. & Fabaceae & $\mathrm{p}$ & $\mathrm{BA} 3$ & subMed \\
\hline 7 & Cachrys alpina M. Bieb. & Apiaceae & $\mathrm{p}$ & $\mathrm{BA} 3$ & Pont \\
\hline 8 & Hyacinthella leucophaea (Koch) Schur & Asparagaceae & $\mathrm{p}$ & & Pont-Med \\
\hline 9 & Iris pumila L. & Iridaceae & $\mathrm{p}$ & & subMed \\
\hline 10 & Morina persica L. & Caprifoliaceae & $\mathrm{p}$ & $\mathrm{BA} 3$ & Med-OT \\
\hline
\end{tabular}




\begin{tabular}{|c|l|c|c|c|c|}
\hline \hline 11 & Paeonia tenuifolia L. & Paeoniaceae & $\mathrm{p}$ & BC1, EN, BA3 & subMed \\
\hline 12 & Prunus fruticosa Pall. & Rosaceae & sh & & Eur-Sib \\
\hline 13 & Ranunculus illyricus L. & Ranunculaceae & $\mathrm{p}$ & & Eur-subMed \\
\hline 14 & Sternbergia colchiciflora Waldst. \& Kit. & Amaryllidaceae & $\mathrm{p}$ & & Eur-Pont \\
\hline
\end{tabular}

Table5. Quaternary postglacial relicts

\begin{tabular}{|c|l|c|c|c|c|}
\hline No & \multicolumn{1}{|c|}{ Species name } & Family & $\begin{array}{c}\text { Biological } \\
\text { type }\end{array}$ & $\begin{array}{c}\text { Conser-vation } \\
\text { status }\end{array}$ & $\begin{array}{c}\text { Floristic } \\
\text { element }\end{array}$ \\
\hline 1 & Lathyrus pancicii (Jurišić) Adamović & Fabaceae & $\mathrm{p}$ & CR, BA2a & Bal \\
\hline 2 & Lathyrus pannonicus (Jacq.) Garcke & Fabaceae & $\mathrm{p}$ & & subMed-Sib \\
\hline 3 & Linum flavum L. & Linaceae & $\mathrm{p}$ & & subMed \\
\hline 4 & Oxytropis pilosa (L.) DC. & Fabaceae & $\mathrm{p}$ & & Eur-CAs \\
\hline 5 & Plantago maxima Juss. ex Jacq. & Plantaginaceae & $\mathrm{p}$ & $\mathrm{BA} 3$ & Eur-As \\
\hline 6 & Rubus saxatilis L. & Rosaceae & $\mathrm{sh}$ & & Eur-As \\
\hline 7 & Viola ambigua Waldst. \& Kit. & Violaceae & $\mathrm{p}$ & & Eur-Sib \\
\hline
\end{tabular}

\section{REFERENCES}

[1] Lovén S. L., Om nagra i Vettern och Venern funna Crustaceer, Öfvers. K. Vetensk. Akad. Förhandl., Stockholm, pp. 285-314 (1862) (in Swedish).

[2] Birshstein Y. A., The term "relic" in biology, Zoologicheski Zhurnal 26(4), 313-330 (1947) (in Russian).

[3] Vulf E. V., The concept of "relict" in botanical geography, Materials on the history of flora and vegetation of USSR, Moscow, Leningrad, pp. 28-56 (1941) (in Russian).

[4] Hampe A. and Jump A. S., Climate Relicts: Past, Present, Future, Annual Review of Ecology, Evolution and Systematics 42, 313-333 (2011).

[5] Habel J. C. and Assmann T. (Eds.), Relict species: Phylogeography and Conservation Biology, Springer-Verlag, Berlin, Heidelberg, p. 442 (2010).

[6] Bhagwat S. A. and Willis K. J., Species persistence in northerly glacial refugia of Europe: a matter of chance or biogeographical traits? Journal of Biogeography 35, 464-482 (2008).

[7] Yordanov D. (Main ed.), Flora of PR Bulgaria, Vol. 1-7, Publishing House of BAS, Sofia (1963-1979) (in Bulgarian).

[8] Velchev V. (Ed.), Flora of PR Bulgaria, Vol. 8-9, Publishing House of BAS, Sofia (1982-1989) (in Bulgarian).

[9] Velchev V. (Ed.), Red Data Book of PR Bulgaria, Vol. 1, Plants, Publishing House of BAS, Sofia, p. 447 (1984) (in Bulgarian).

[10] Peev D., Kozuharov St., Anchev M., Petrova A., Ivanova D. and Tzoneva S., Biodiversity of Vascular Plants in Bulgaria, In: Curt Meine (Ed.), Bulgaria's Biological Diversity: Conservation Status and Needs Assessment, Volumes I and II, Washington, D.C., Biodiversity Support Program (1998).

[11] Peev D. (Main ed.), Red Data Book of Republic of Bulgaria. Vol. 1. Plants and Fungi, IBER BAS \& MEW, Sofia, p. 888 (2011) (in Bulgarian).

[12] Biserkov V. (Main ed.), Red Data Book of Republic of Bulgaria, Vol. 3. Natural habitats, IBER - BAS \& MEW, Sofia, p. 458 (2011) (in Bulgarian).

[13] Peev D., Petrova A., Apostolova I. and Asyov B. (Eds.), Important Plant Areas in Bulgaria, PENSOFT, Sofia-Moscow, p. 469 (2012) (in Bulgarian).

[14] Peev D., National park Rila. Management plan 2001-2010, Adopted by Resolution №522 of Council of Ministers on 04.07.2001, Sofia, p. 337 (2001) (in Bulgarian).

[15] Asyov B., Goranova V. and Stoykov D., Flora and fungal diversity of Vrachanski Balkan Natural Park, In: Management plan of Vrachanski Balkan Natural Park, Adopted by Resolution №570 of Council of Ministers on 14.10.2011, Agrolesproject Ltd., Sofia, p. 477 (2011) (in Bulgarian).

[16] Bozhilova E., Koeva Y. and Tonkov S., Systematics of vascular plants, St. Kliment Ohridski Univ. Publ., Sofia, p. 320 (1992) (in Bulgarian).

[17] Gruev B. and Kuzmanov B., General biogeography, St. Kliment Ohridski Univ. Publ., Sofia, p. 498 (1994) (in Bulgarian). 
[18] Petrova A., A contribution to the flora of East Bulgaria, Phytologia Balcanica 10(2-3), 201-205 (2004).

[19] Tzonev R. and Karakiev T., Plantago maxima (Plantaginaceae): a relict species new for the Bulgarian flora, Phytologia Balcanica 13(3), 347-350 (2007).

[20] Uzunova S. and Uzunov S., The plants in Strandzha Natural Park, State Forestry Agency, Directorate of Strandzha Natural Park, p. 215 (2008) (in Bulgarian).

[21] Bozukov V. S. and Tsenov B. V., Catalogue of the Cenozoic plants of Bulgaria (Eocene to Pliocene). Addendum and Corrigendum, Phytologia Balcanica 18(3), 237-261 (2012).

[22] Tashev A. N., Conservation importance of the dendroflora of the Pirin Mountain (Bulgaria), Phytodiversity of Eastern Europe 8(4), 4-13 (2014).

[23] Domin K., Some Remarks on the Genesis of the Flora of Southern Slovakia, Berichte der Schweizerischen Botanischen Gesellschaft 46, 545-555 (1936).

[24] Panetsos C. P., Natural hybridization between Pinus halepensis and Pinus brutia in Greece, Silvae Genetica 24(5-6), 163-168 (1975).

[25] Fésüs I., Interaction Between Agriculture and Environment in Hungary, IUCN, Information Press, Oxford, p. 113 (1992).

[26] Schlee M., Grimm G., Göker M., Sauer W. and Hemleben V., Molecular and phytosociological insight into postglacial relicts Lathyrus pannonicus and Oxytropis pilosa In: Habel J. (Ed.), Phytogeography and Conservation of Postglacial Relicts, Book of Abstracts, Luxembourg, pp. 31-32 (1997).

[27] Bajzáth J., Plant macrofossils from Hungarian Pleistocene III. Palaeobotanical study in Győrújfalu, Western Hungary, Annals Historico-Naturalis Musei Nationalis Hungarici 90, 17-26 (1998).

[28] Denk T., Frotzler N. and Davitashvili N., Vegetational patterns and distribution of relict taxa in humid temperate forests and wetlands of Georgia (Transcaucasia), Biological Journal of the Linnean Society 72, 287-332 (2001).

[29] Galdean N., Needs assessment report for the Natural Park Iron Gates, Regional environmental center for Central and Eastern Europe local office Bucharest, Romania, p. 56 (2002).

[30] Boža P., Anačkov G., Igić R., Vukov D. and Polić D., Flora "Rimskog šanca" (Vojvodina, Srbija), 8th Symposium on the flora of Southeastern Serbia an Neighbouring Regions, Niš, 2024.06.2005, Abstracts, p. 55 (2005).

[31] Demeter L., Pásztohy Z., Cserg A.-M. and Cărăuş I., Terrace ponds in the Ciuc Basin: a preliminary characterization, Universitatea din Bacău, Studii şi Cercetări, Biologie 10, 19-23 (2005).

[32] Thompson J. D., Plant Evolution in the Mediterranean, Oxford University Press, p. 293 (2005).

[33] Andonovski V. and Pop-Stojanov D., Feasibility Study for the establishment of the National Park in the Macedonian side of Shara Mountain, Balkan Foundation for Sustainable Development, Skopje, p. 23 (2006).

[34] Drescher A., Prots B. and Yena A. V., Notes on Crimean Flora (botanical excursion on the Crimean peninsula), FRITSCHIANA, Veröffentlichungen aus dem Institut für Pflanzenwissenschaften, Bereich Systematische Botanik und Geobotanik, der Karl-FranzensUniversität Graz, p. 71 (2007).

[35] Lukash O., Distribution, cenotic characteristic and protection of habitats of plants of the Bern Convention in East Polesye, Thaiszia-Journal of Botany Košice 17, 33-58 (2007).

[36] Nikolić L., Stojanov S. and Ranđelović N., The endemic and relict plants species in the valley of the River Pčinja, In: Trumić, M. (ed.), Proceedings Ecological Truth, Serbia, pp. 34-39 (2007).

[37] Obratov-Petkovic D., Popovic I. and Dajic-Stevanovic Z., Diversity of the vascular flora of Mt. Zlatar (Southwest Serbia), EurAsian Journal of BioSciences 5, 35-47 (2007).

[38] Avramović D., Nikolić L., Zlatković B. and Ranđelović N., The proposal of rare plant species of the valley of river Pčinja nominated for protection, Proceedings from 3 Congress of Ecologist of Macedonia with international participation and marking the 80-Anniversary of Prof. dr Ljupčo Grupče's life and 60 years active scientific work, Macedonian Ecological Society, Skopje, pp. 182-186 (2008). 
[39] Rakaj M., Floristic and chorological news from North Albania, Botanica Serbica 33(2), 177-183 (2009).

[40] Stevanović V., Vukojičić S., Šinžar-Sekulić J., Lazarević M., Tomović G. and Tan K., Distribution and diversity of Arctic-Alpine species in the Balkans, Plant Systematics and Evolution 283(3-4), 219-235 (2009).

[41] Jakšić P. and Momirović M., Contribution to understanding the origin and the genesis of the Nišava riverside valley fauna, Biologica Nyssana 1(1-2), 123-130 (2010).

[42] Oprea A., Goia I., Tănase C. and Sîrbu C., Assessment of species composition: endemics, relicts and red-listed plants (tracheophytae, bryophytae and fungi) in forest natural habitats of Romania, Contribuţii Botanice XLV, pp. 13-24 (2010).

[43] Stanković M., Rarely, threatened and relict species in flora NPA "Zasavica", 10th Symposium on the Flora of Southeastern Serbia and Neighbouring regions, Vlasina, 17-20 June 2010, Abstracts, pp. 66-67 (2010).

[44] Murvanidze M., Mumladze L., Arabuli T. and Kvavadze E., Landscape distribution of oribatid mites (Acari, Oribatida) in Kolkheti National Park (Georgia, Caucasus), In: Moraes, G. J., H. Proctor (eds), Acarology XIII: Proceedings of the International Congress, Zoosymposia 6, 221233 (2011).

[45] Bank P., Bemmerlein-Lux F., Gagica I. and Hajredini E., Sustainable Development Atlas, Framework for a comprehensive and balanced rural development for the Municipality of Dragash / Dragaš Kosovo, Volume V: Data, Dragaš, Kosovo, p. 97 (2012).

[46] Kaplan Z., Flora and Phytogeography of the Czech Republic, Preslia 84, 505-573 (2012).

[47] Oprea A. and Sîrbu C., The vascular flora of Rarău Massif (Eastern Carpathians, Romania). Note I, Memoirs of the Scientific Sections of the Romanian Academy, Vol. XXXV, pp. 55-84 (2012).

[48] Horatu P. E., Study on ecotourism development in transboundary region of Djerdap National Park and Porțile de Fier Nature Park in frame of Workpackage no. 6 of the Bioregio Carpathians Project, Final report, p. 110 (2014).

[49] Valeriu-Ioan V., Contributions to the study of flora in the Orăştie River BASIN (CentralWestern Romania), Analele Universităţii din Oradea, Fascicula Protecţia Mediului, Vol. XXII, pp. 103-110 (2014).

[50] Velitzelos D., Bouchal J. M. and Denk T., Review of the Cenozoic floras and vegetation of Greece, Review of Palaeobotany and Palynology 204, 56-117 (2014).

[51] Angiosperm Phylogeny Group, An update of the Angiosperm Phylogeny Group classification for the orders and families of flowering plants: APG III, Botanical Journal of the Linnean Society 161(2), 105-121 (2009).

[52] Bern Convention, Convention on the Conservation of European Wildlife and Natural Habitats, Appendix I, 2002, http://conventions.coe.int/Treaty/FR/Treaties/Html/104-1.htm (accessed 30 December 2015).

[53] Convention on International Trade in Endangered Species of Wild Fauna and Flora, Appendix II, 2009, http://www.cites.org/eng/app/appendices.shtml (accessed 30 December 2015).

[54] Directive 92/43/EEC, Council Directive 92/43/EEC of 21 May 1992 on the Conservation of Natural Habitats and of Wild Fauna and Flora, Appendix II, OJ L 206, 22.07.1992.

[55] Biodiversity Act of Republic of Bulgaria, State Gazette number 77, 9 August 2002, pp. 9-42. Last amended in State Gazette number 27, 15 March 2013 (in Bulgarian).

\section{AUTHOR's BIOGRAPHY}

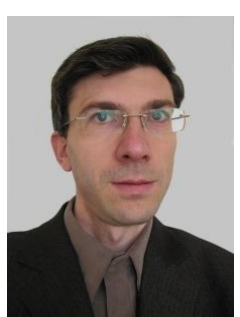

Dimcho Zahariev, is currently an Associated Professor in the Faculty of Natural Sciences, University of Shumen Bishop Konstantin Preslavski, Shumen, Bulgaria. $\mathrm{He}$ is $\mathrm{PhD}$ in biology (Botany scientific specialty). He teaches Anatomy and Morphology of Plants, Plant Systematics, Phytogeography and conservation of the biological diversity, Floristic diversity in Bulgaria. His research interests are in: Floristry, Medicinal plants and Biodiversity. Member of the Bulgarian Phytosociological Association 